\title{
ANGULAR DEPENDENCE OF HeNe-LASER LIGHT SCATTERING BY BOVINE AND HUMAN DENTINE
}

\author{
J. R. ZIJP and J. J. TEN Bosch \\ Laboratory for Materia Technica, State University of Groningen, A. Deusinglaan 1, \\ 9713 AV Groningen, The Netherlands
}

(Received 20 April 1990; accepted 30 November 1990)

\begin{abstract}
Summary-The scattering phase functions for HeNe-laser light of dentine sections 10-20 $\mu \mathrm{m}$ thick were measured. The functions perpendicular to the tubules had first-order maxima at angles of $4^{\circ}$ for bovine dentine and $5^{\circ}$ for human dentine; those parallel to the tubules showed no first-order maxima. Several corrections were made before the asymmetry factors $g$ for intertubular dentine were determined; the average values are for bovine dentine $g=0.37$ and for human dentine $g=0.44$.
\end{abstract}

Key words: lasers, optical properties, light-scattering, dentine human and bovine.

\section{INTRODUCTION}

The behaviour of light in dentine may be examined in order to match prosthetic materials to natural dentine, and to assess if optical measurements can provide an indirect measure of dentine caries.

The optical matching of restorative and prosthetic materials has been by trial, error and experience, with both very good and very bad results (van der Burgt et al., 1985). Better matching may be achieved by a better understanding of the optical processes in natural tissues. In both dental enamel and dentine, light scattering is the most important determinant of colour and appearance. In enamel the hydroxyapatite crystals are mainly responsible for this (Spitzer and ten Bosch, 1975). It is already possible to quantify enamel caries by measuring light scattering (ten Bosch, van der Mei and Borsboom, 1984). In dentine the tubules are the most important scatterers (ten Bosch and Zijp, 1987). When dentine is decalcified by caries, the cross-section of the dentinal tubules is increased (Arends, Ruben and Jongebloed, 1989). This causes a change in the scattering properties, which in the future might be used to quantify dentine caries.

The absorption $(K)$ and scattering $(S)$ coefficients for diffuse light in dentine have been recorded by ten Bosch and Zijp (1987). We have now measured the angular dependence of the light scattering and the anisotropy factor $g$.

\section{THEORY}

The colour of all materials is determined by the diffuse absorption coefficient $K$, the diffuse scattering coefficient $S$ and the phase function $I(\theta)$ of the scatterers. In translucent materials like dental enamel and dentine the light penetrates the material before it re-emerges. The absorption and therefore the colour is exponentially dependent on the penetration path. The thickness of teeth at the edges is about the same order of length as the real free path of the photons.
So the phase functions, which describe the intensity of the scattered light as a function of the scattering angle, have to be known for good colour matching. In general the phase functions depend on the directions of polarization of both the incident and the scattered light. In most calculations the asymmetry factor $g$, the mean cosine of the scattering angle of all photons, is used to estimate the penetration path length. $g$ can be determined by:

$$
g \equiv \frac{\int_{0}^{180} \int_{0}^{360} I(\theta, \varphi) \cos (\theta) \mathrm{d}(\varphi) \mathrm{d}(\theta)}{\int_{0}^{180} \int_{0}^{360} I(\theta, \varphi) \mathrm{d}(\varphi) \mathrm{d}(\theta)}
$$

When the scattering phase function is circularly symmetrical round the axis of the incident beam:

$$
g=\frac{\int_{0}^{180} I(\theta) \cos (\theta) \sin (\theta) \mathrm{d}(\theta)}{\int_{0}^{180} I(\theta) \cos (\theta) \mathrm{d}(\theta)} .
$$

For low $g$ values $(g \approx 0)$ the scattering process is isotropic and a photon re-emerges at the surface after a short path and contributes to volume reflection. For high $g$ values $(g \approx 1)$ the scattering is forward peaked, so that light paths are long and there is a relatively high probability of absorption. The linear absorption coefficient $a$ will be neglected in this paper, because in dentine $a=K / 2$ is small compared to $S . S$ can be converted to the scattering coefficient for collimated beams $s$ by $s=(4 S+a) / 3(1-g) \approx 4 S / 3(1-g)$ for $a \ll 4 S$ (Meador and Weaver, 1979). We define the effective mean free path $S^{-1}$, which is the mean distance covered by a photon before it is backscattered, and the real mean free path $s^{-1}$, which is the mean distance that a photon covers between two collisions with scatterers.

In dentine sections, as schematically drawn in Fig. 1, scattering can be split up into three distinct 


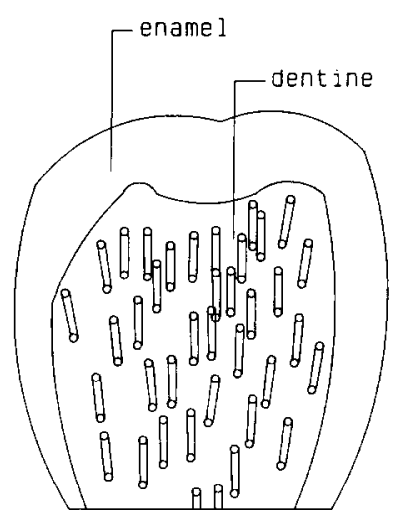

Fig. 1. Schematic outline of a dentine section, tubules not to scale.

processes. Firstly a rotation-symmetrical process (round the principal axis) caused by surface roughness of the sections. Secondly, a rotation-symmetrical process caused by stochastic inhomogeneities in density. Thirdly a rotation asymmetrical process caused by the presence of the tubules which are oriented from the pulp to the dentine-enamel junction, and so are lying parallel when a small area is observed. If the tubules are considered as infinitely long, straight cylinders, they will scatter only in the plane perpendicular to their axes (van de Hulst, 1957).

When a thin section is observed in a transmission light microscope, it is apparent that it can be considered as a periodic grating of transparent intertubular dentine between oblique rods, i.e. the tubules surrounded by peritubular dentine. Such a grating will cause Fraunhofer diffraction, the spacing $d$ between the tubules follows from $d=n \lambda / \sin (\theta)$, in which $n=1.45$ (ten Bosch and Zijp, 1987) is the index of refraction of intertubular dentine. Fraunhofer diffraction was also observed in human dental enamel by O'Brien (1988), caused by the periodical presence of slits between the hydroxyapatite prisms.

\section{MATERIALS AND METHODS}

From the buccal side of two freshly extracted bovine incisors and six human third molars, $80 \mu \mathrm{m}$ sections of dentine were cut, parallel to the buccal surface. These 10 and 34 sections, respectively, were stuck into a $10 \mu \mathrm{m}$ deep groove in a metal disc, sanded down to $20 \mu \mathrm{m}$ on 1200 -mesh sanding paper, and ultrasonically cleaned in water to reduce the smear layer. The sections were stored on wetted tissues until measurement.

The measurement equipment is schematically outlined in Fig. 2. An incident beam, supplied by a $5 \mathrm{~mW}$ HeNe-laser $(\lambda=632.8 \mathrm{~nm})$ polarized $45^{\circ}$ with respect to the vertical, enters a glass cell filled with milliporefiltered water (Millex-GV $0.22 \mu \mathrm{m}$, Millipore S.A., Molsheim, France). For scattering angles from $168^{\circ}$ to $20^{\circ}$ we used a round cell (type $540.114-\mathrm{OS} 25 \mathrm{~mm}$ HELLMA, Mullheim/Baden, Germany), because at all these angles the water-glass and the glass-air interface are perpendicular to the scattered light, and so the reflections at the interfaces are minimal. The incident beam is convergent after entering the round cell. This will mainly disturb the measurement at low scattering angles. Therefore at scattering angles of $20^{\circ}-0^{\circ}$ we used a square cell (type 101.062-OS $20 \mathrm{~mm}$ ). At these angles a neutral density filter was placed in the incident beam to prevent saturation of the detection system. The measured phase functions at these angles were corrected for the index of refraction transition at the water-glass and the glass-air junctions, which cause enlarging of the observed scattering angle in the air and the angle of acceptance of the detector with respect to these angles in water.

A T-shaped holder, painted black to prevent forward scattered light from being reflected backward by the cylindrical cell wall, was placed in the centre of the scattering cell. The dentine section was mounted on this holder, and adjusted so that no scattering from scratches was observed. The section was placed in water to achieve low surface reflections at the water-dentine junctions.

The centre of the section was focused with a single $57 \mathrm{~mm}$ lens on a photodiode mounted at a turnable bar $405 \mathrm{~mm}$ from the centre of the cell; the position of the bar and the measured intensity were read by a computer. A red filter was used to prevent the blue gas-discharging light from reaching the detector. The steric angle of observation was sufficiently large at $8 \times 10^{-5}$ sr to smooth speckle patterns. Eight readings were taken for each sample: the polarization filter in front of the detector horizontal and vertical, at high and low scattering angles (with the round and the square scattering cell, respectively) and in the plane of and perpendicular to the tubule axes.

Like Jacques et al. (1987), we normalized the measured phase functions in the plane of the tubules to $2 \pi$. We further adopted their principle of plotting but did not adopt an isotropic scattering function to account for backward scattering. Thus, we plotted $I(\theta)^{-3 / 2}$ as a function of $\cos (\theta)$. In the interval $0.7<\cos (\theta)<0.92$ this plot appeared linear, indicating that $I(\theta)$ resembled a Henyey-Greenstein phase function (Henyey and Greenstein, 1941). We extrapolated the linearity towards $\cos (\theta)=1.0$, which gave calculated $I(\theta)$ values in this $\theta$ interval including $I\left(0^{\circ}\right)$. In the interval of $\theta \approx 90^{\circ}$, we assumed the phase functions to be linear and equal to the measured values at the borders of the interval, as indicated by the dotted lines in Figs 4(a) and 5(a). In the interval $\theta \approx 180^{\circ}$ we assumed the phase functions to be constant and equal to the value at $168^{\circ}$. After these corrections we calculated the anisotropy factor $g$ by formula (2).

The transmission of the sections at $\theta=0^{\circ}$ was measured. The contribution of $I\left(0^{\circ}\right)$ to this transmission was very small and neglected, as were reflections at the dentine-water interface. The mean thickness of each section was calculated from micrometer measurements at five different points.

The phase functions of two monodispersed polystyrene latex suspensions ( $\phi 688$ and $2010 \mathrm{~nm}$ ) were measured to check the sensitivity of the equipment. These suspensions were diluted to a mass concentration of $1.6 \times 10^{-6}$ solid to guarantee single scattering in the cell (Harris, Sherman and Morse, 1967). 

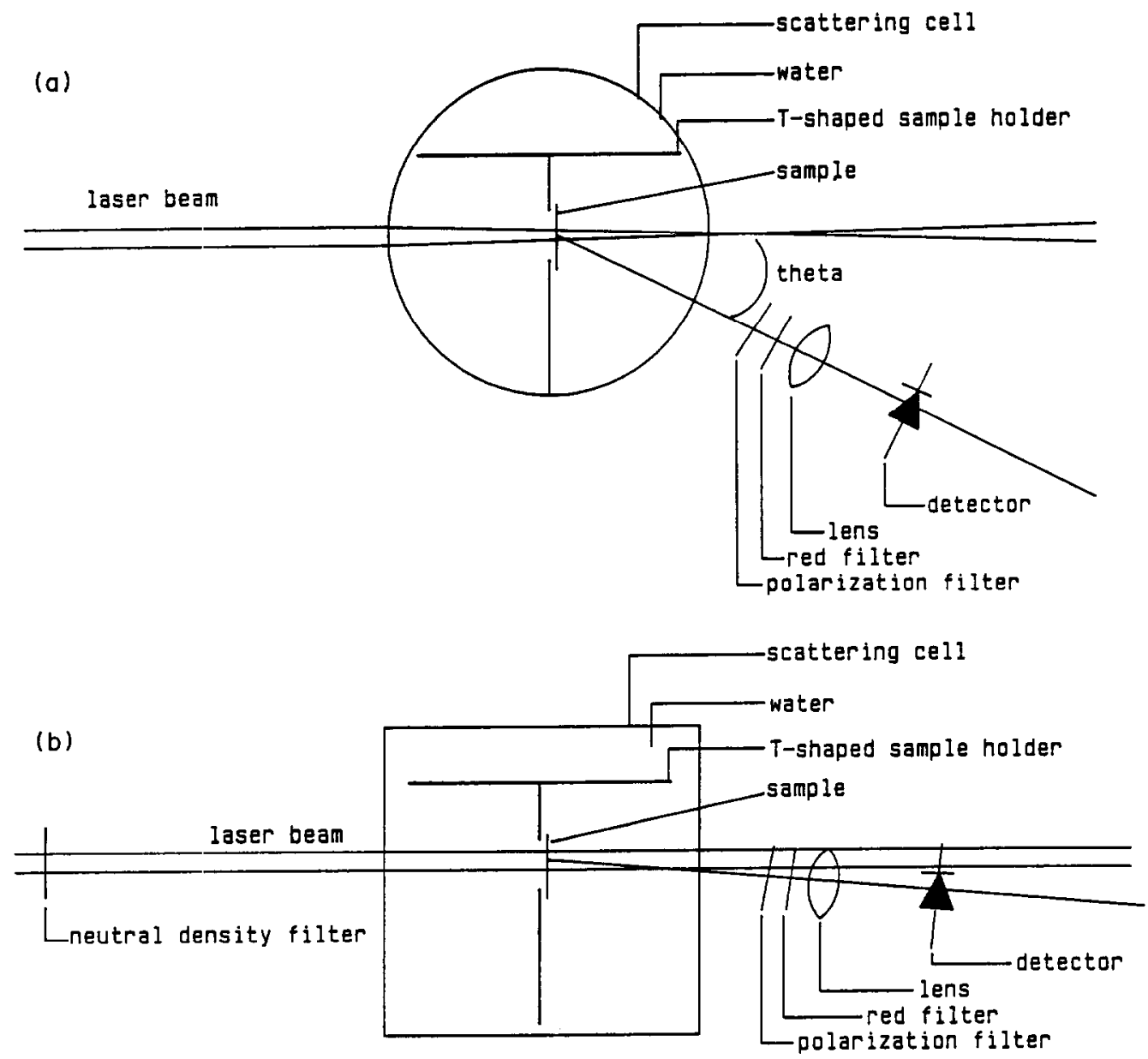

Fig. 2. Schematic outline of the measurement equipment. (a) For angles $168^{\circ}-20^{\circ}$. (b) For angles $20^{\circ}-0^{\circ}$.

All values shown are means with their standard deviation.

\section{RESULTS}

The measured and theoretical single scattering phase functions of $2010 \mathrm{~nm}$ latex spheres in water are shown in Fig. 3. In Fig. 3(a) the light is polarized perpendicular to the plane of scattering (vertical); in Fig. 3(b) the light is polarized in the plane of scattering (horizontal); in both figures the intensity axis is logarithmic.

In Fig. 4 the measured phase functions of a typical bovine dentine sample are shown: in Fig. 4(a) with the tubules oriented parallel to the plane of scattering; in Fig. 4(b) with the tubules perpendicular to the plane of scattering. Figures 5(a) and (b) show the phase functions of a typical human sample. These do not depend on the orientation of the polarization filter in front of the detector. The functions are strongly forward-peaked. In the plane perpendicular to the tubules there is a first-order maximum for bovine dentine at $4^{\circ}\left( \pm 0.8^{\circ}\right)$, and for human dentine at $5^{\circ}\left( \pm 0.6^{\circ}\right)$. The $g$ values determined from the corrected phase functions in the plane of the tubules are shown in Table 1. Table 2 gives an overview of the optical properties of dentine at
$633 \mathrm{~nm}$.

The measured collimated transmission of most sections at $\theta=0^{\circ}$ is plotted as a function of the sample thickness in Fig. 6.

\section{DISCUSSION}

Comparison of the measured and the calculated scattering phase functions of the $2010 \mathrm{~nm}$ polysterene latex spheres shows agreement in form near $90^{\circ}$; the deviations at lower and higher scattering angles are probably caused by internal reflections and enlarging of the cross-section of the observation beam and the incident beam. This indicates that our equipment is sensitive enough to measure the phase functions of single light scatterers. The measured phase functions of $688 \mathrm{~nm}$ spheres showed similar deviations.

As our samples are made as thin as possible, the effect of multiple scattering will be small. The effective mean free path can be estimated by using $S=8 \mathrm{~mm}^{-1}$ at $633 \mathrm{~nm}$ (ten Bosch and Zijp, 1987), $S^{-1}=1 / 8 \mathrm{~mm}=0.13 \mathrm{~mm}$, which is long compared to the thickness of the samples. Taking a mean value for $g=0.4$ (Table 2), the real mean free path can be estimated at $0.011 \mathrm{~mm}$, a value of the order of the sample thickness. 
$I(\theta)$

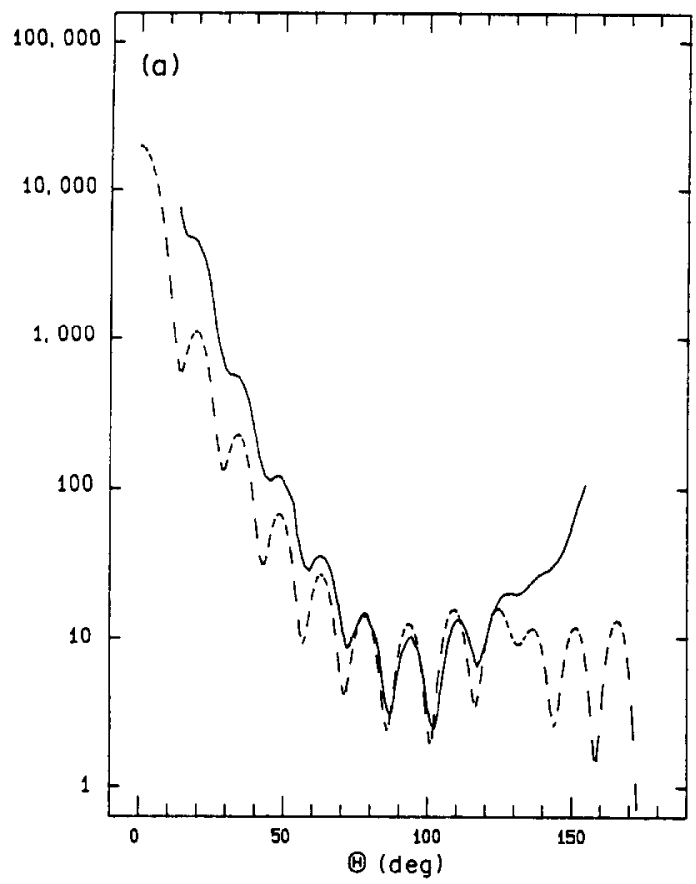

$I(\theta)$

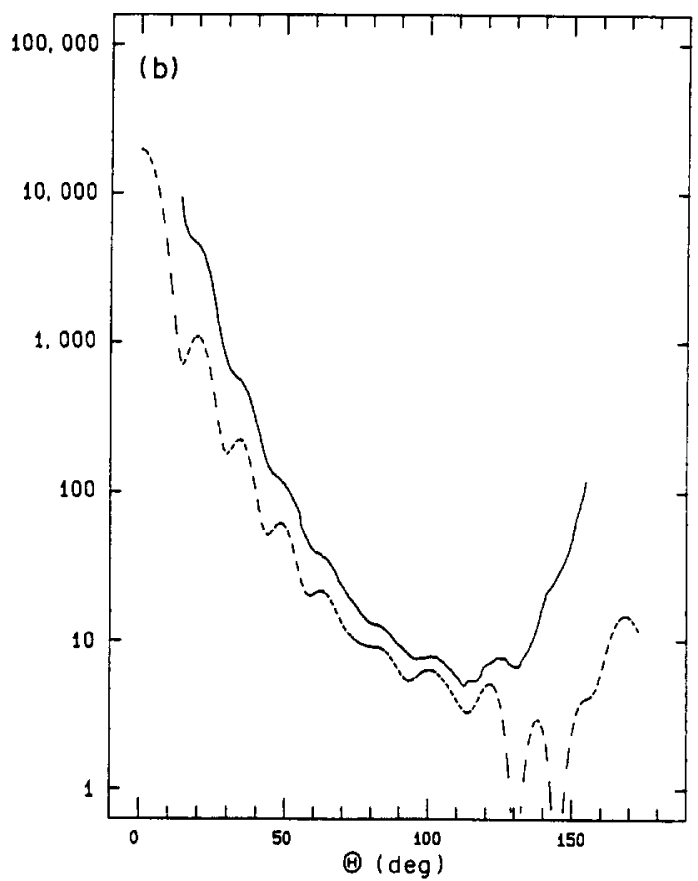

Fig. 3. Comparison of calculated and measured phase functions of $2010 \mathrm{~nm}$ polystyrene latex spheres in watcr. (a) Polarization perpendicular to plane of scattering. (b) Polarization parallel to plane of scattering. Full lines: measured; interrupted lines: theoretical.

\section{The phase functions}

The phase functions as shown in Figs 4 and 5 have a minimum around $90^{\circ}$, possibly due to the

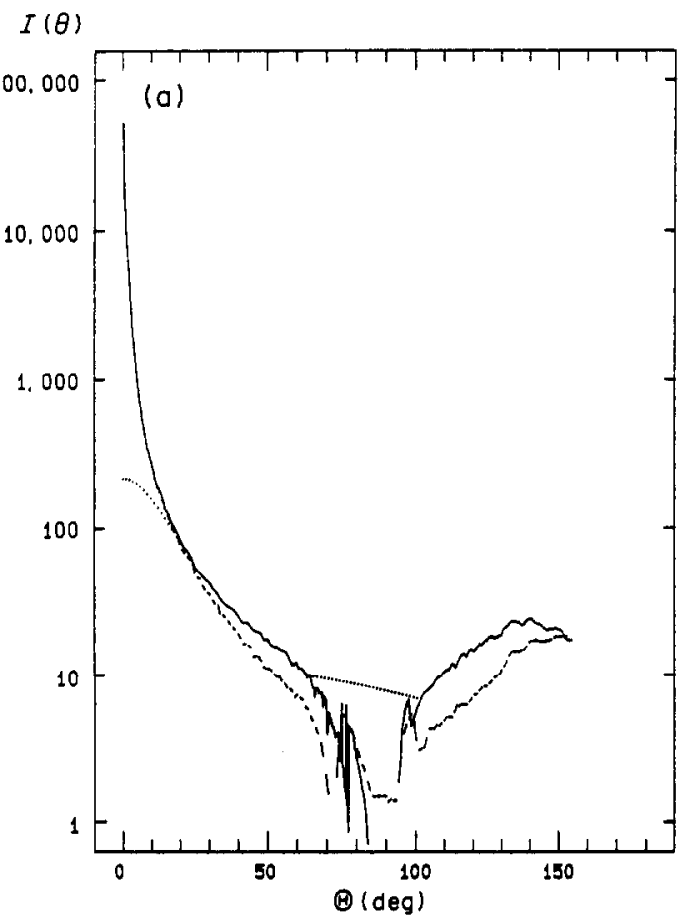

shadow of the T-shaped sample holder, or to multiple scattering, because at this angle the light path through the material is much longer than the intertubular distance. In shape as well as in the

$$
I(\theta)
$$

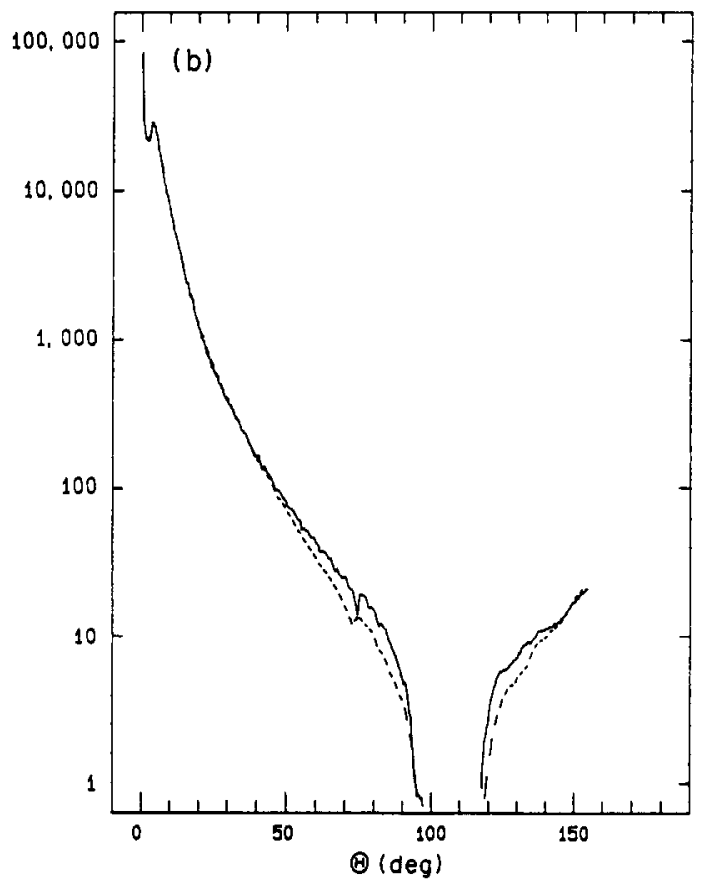

Fig. 4. Measured (unnormalized) phase functions of bovine dentine with tubules parallel (a) and perpendicular (b) to plane of scattering. Full and interrupted lines: polarization perpendicular and parallel to this plane, respectively. Dotted lines: correction for sample holder $\left(\approx 90^{\circ}\right)$ and Henyey-Greenstein function approximation $\left(\approx 0^{\circ}\right)$. 
$I(\theta)$

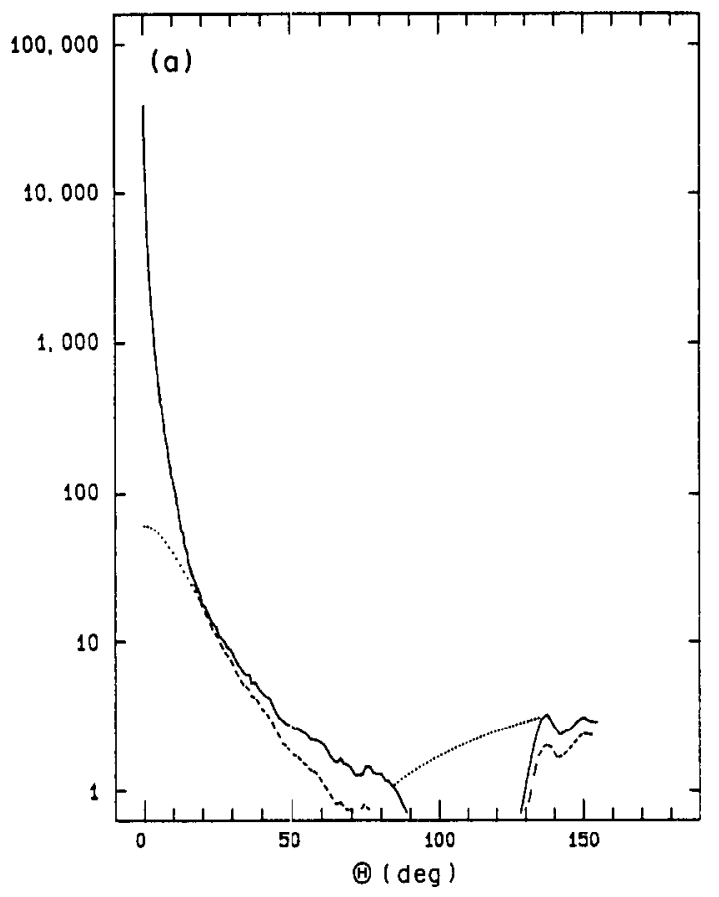

$I(\theta)$

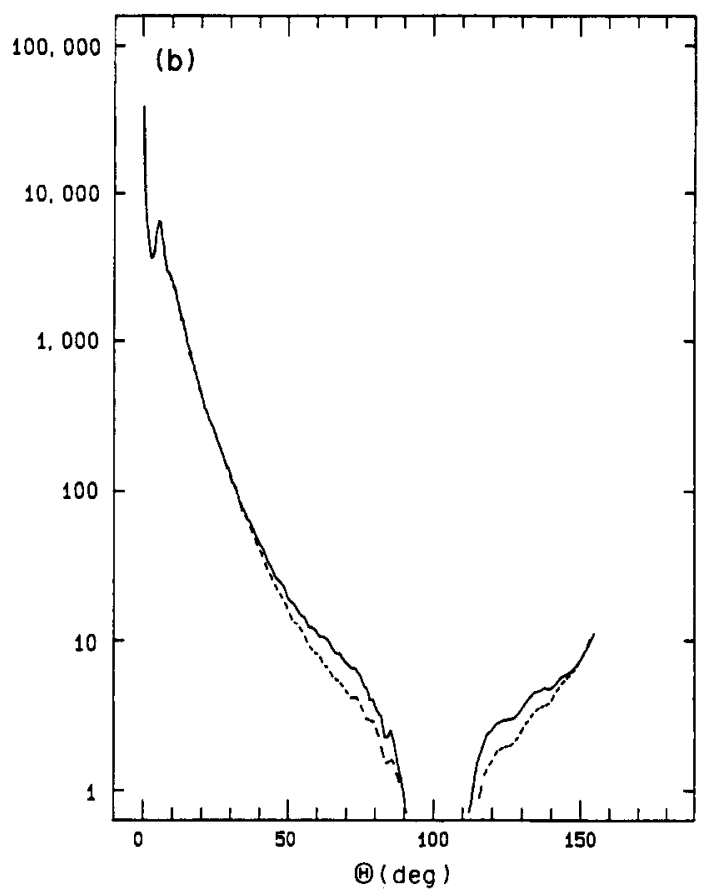

Fig. 5. As Fig. 4, but for human dentine.

Table 1. $g$ Values and the angles of first-order maxima

\begin{tabular}{lccccc} 
& \multicolumn{2}{c}{$g$ Values determined } & & \multicolumn{2}{c}{$\begin{array}{c}\text { Angle of first-order } \\
\text { maxima }\left(^{\circ}\right)\end{array}$} \\
\hline Polarization & Bovine $n=10$ & Human $n=34$ & & Bovine & Human \\
\hline Horizontal & $0.32 \pm 0.08$ & $0.39 \pm 0.07$ & & $4.0 \pm 0.8$ & $5.0 \pm 0.6$ \\
Vertical & $0.41 \pm 0.09$ & $0.48 \pm 0.08$ & & & \\
\hline
\end{tabular}

Mean values \pm standard deviations.

location of the minimum and the ratio between maximum and minimum intensity, these curves resemble those of other tissues (Marchesini et al, 1989).

The species of the sample cannot be deduced from the form of its phase function, because of the very large intra-species differences.

The curves in Fig: 4(b) and 5(b) are higher than those in Figs 4(a) and 5(a) for angles in the region $3^{\circ}-90^{\circ}$ and so the main part of the intensity is scattered perpendicular to the tubules. This is another demonstration that the tubules are the main scatterers. The first-order maxima of the measured phase functions in the plane perpendicular to the tubules can be explained in two ways. Firstly, maxima in the single scattering-phase functions of the tubules may cause similar maxima for thin sections ( $\mathrm{Zijp}$ and ten Bosch, 1991). Secondly, they may be due to Fraunhofer diffraction. This would suggest an intertubular spacing of $6 \mu \mathrm{m}$ for bovine dentine, and $5 \mu \mathrm{m}$ for human dentine, both lower than the 10 and $6 \mu \mathrm{m}$ intertubular distances measured on microphotographs of several other samples. The lack of secondorder maxima may result from lack of constancy in the intertubular distance, the variations in plane of the tubules, and the variations from exact parallelism of the tubules. In view of these complications and possible coherence effects we did not investigate this further.

\section{The $g$ values}

From Table 1 we see that the $g$ values do not depend on the orientation of the polarization filter in front of the detector. We expected this because Figs 4 and 5 do not show any principal difference between these phase functions.

The $g$ values we found are much lower than those found for human blood $(0.974)$ and several soft tissues (chicken muscle $0.965 \pm 0.004$, bovine muscle $0.954 \pm 0.016$, and pig brain $0.940 \pm 0.029$ ) (Flock,

Table 2. Optical properties of dental materials at $633 \mathrm{~nm}$

\begin{tabular}{lccc}
\hline Material & $\begin{array}{c}\text { Scattering } \\
s\left(\mathrm{~mm}^{-1}\right)\end{array}$ & $\begin{array}{c}\text { Absorption } \\
a\left(\mathrm{~mm}^{-1}\right)\end{array}$ & $\begin{array}{c}\text { Anisotropy } \\
g\end{array}$ \\
\hline Bovine dentine & $200^{1}$ & $0.35^{2}$ & $0.36^{3}$ \\
Human dentine & $120^{1}$ & $0.60^{2}$ & $0.44^{3}$ \\
Bovine enamel & $0.054^{4}$ & $0.097^{4}$ & - \\
Human enamel & $0.11^{4}$ & $0.097^{4}$ & - \\
\hline
\end{tabular}

IThis work.

${ }^{2}$ From $K$ value cited in ten Bosch and Zijp (1987) $a=K / 2$.

${ }^{3}$ This work, average of values for perpendicular and horizontal polarization.

${ }^{4}$ From $s_{\mathrm{c}}$ and $a_{\mathrm{c}}$ cited in Spitzer and ten Bosch (1975). 


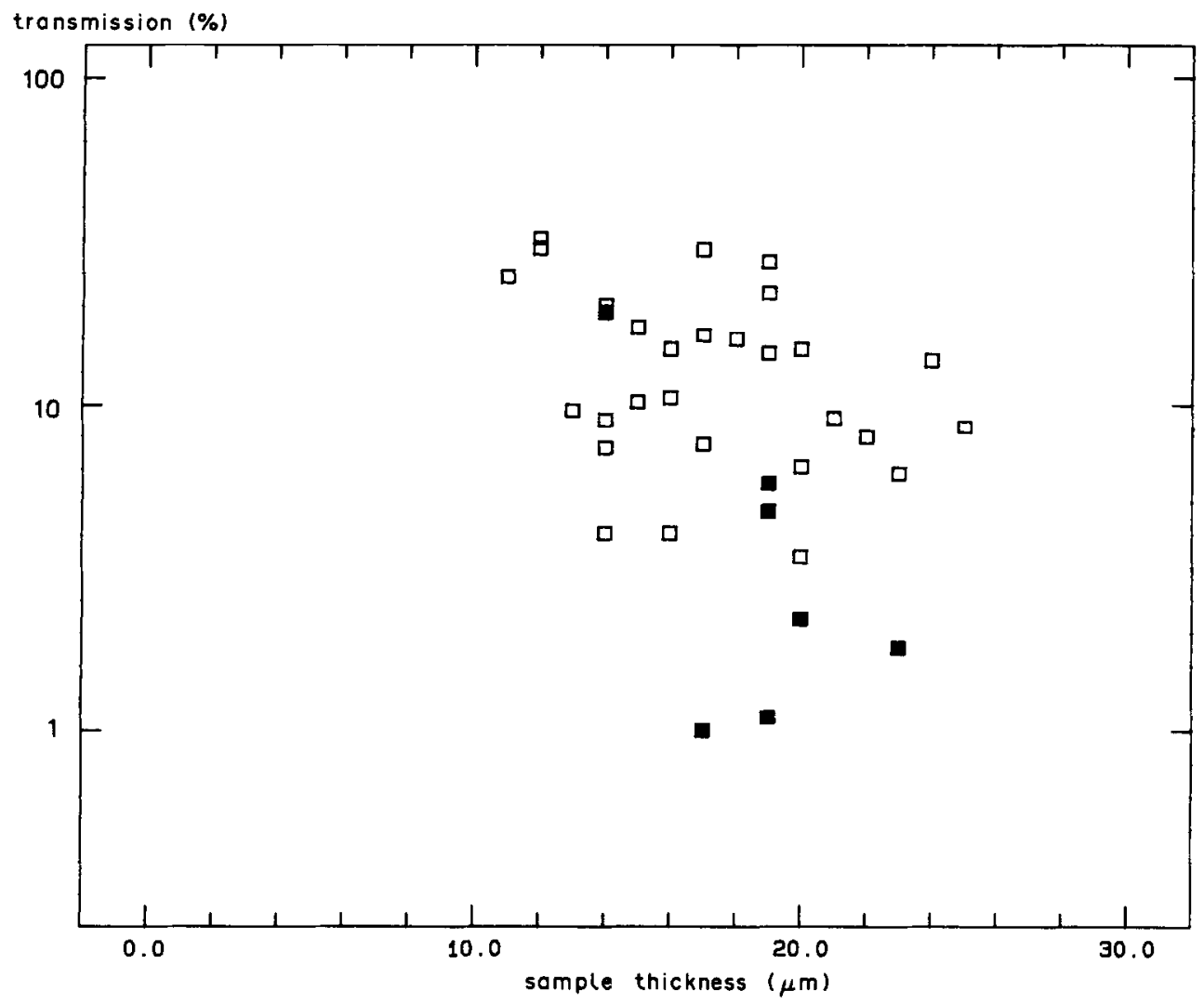

Fig. 6. Transmission at $\theta=0^{\circ}$ of the samples as a function of sample thickness. Bovine dentine.

$\square$ Human dentine.

Wilson and Patterson, 1987) and human dermis (0.82) (Jacques, Alter and Prahl, 1987). For good colour matching we recommend prosthetic dentine materials having a $g$ value between 0.32 and 0.48

\section{The transmission at $\theta=\theta^{\circ}$}

Figure 6 demonstrates that there is no strong relation between the sample thickness and the transmission of the sections at $\theta=0^{\circ}$. No relation was expected because for thin sections the biological variations in tubule density and tubule cross-section affect the transmission more than the sample thickness. Nevertheless, an average linear scattering coefficient $s$ can be estimated from these values using the Lambert-Beer law: $T=\mathrm{e}^{-s d}$. For the bovine dentine samples the mean thickness is $18.7 \mu \mathrm{m}$ and the mean value of $\ln (T)$ is -3.50 . Thus $s=200 \mathrm{~mm}^{-1}$ and so $S=3 \times s(1-g) / 4=89 \mathrm{~mm}^{-1}$, which is much higher than our previous published values of $14-26 \mathrm{~mm}^{-1}$ (ten Bosch and Zijp, 1987). For human dentine the mean value of the sample thickness is $17.3 \mu \mathrm{m}$ and the mean value of $\ln (T)$ is -2.14 . Thus $s=120 \mathrm{~mm}^{-1}$ and so $S=3 \times s(1-\mathrm{g}) / 4=52 \mathrm{~mm}^{-1}$, which is also much higher than our previous published values of $3-8 \mathrm{~mm}^{-1}$ (ten Bosch and Zijp, 1987). The $s$ values compare favourably with values for soft tissue (e.g. Marchesini et al., 1989; Parsa, Jacques and Nishioka, 1989), which range $15-50 \mathrm{~mm}^{-1}$. As dentine is a mineralized tissue with larger internal differences in refractive index, $s$ values higher by a factor $<10$ are not surprising.
The discrepancy in $S$ values probably arises from our earlier assumption that the incoming light was diffuse (which it was not) or that the scattering was diffuse $(g=0)$, which now appears wrong (Table 1). Further work may be desirable to confirm these new values.

We plan to make measurements on optically flat samples where roughness does not contribute to light scattering, in the hope of distinguishing between inhomogeneity scattering and scattering from tubules.

Acknowledgement-The authors thank $\mathrm{R}$. Graaff from the department of Obstetrics and Gynaecology for helpful discussions and critical reading of the manuscript.

\section{REFERENCES}

Arends J., Ruben J. and Jongebloed W. J. (1989) Dentine caries in vivo. Caries Res. 23, 36-41.

ten Bosch J. J. and Zijp J. R. (1987) Optical properties of dentin. In Dentine and Dentine Reactions in the Oral Cavity (Eds Thylstrup A., Leach S. A. and Qvist V.), Chap. II, pp. 59-65. IRL Press, Oxford.

ten Bosch J. J., van der Mei H. C. and Borsboom P. C. F. (1984) Optical monitor of in vitro caries. Caries Res. 18, 540-547.

van der Burgt T. P., ten Bosch J. J., Borsboom P. C. F. and Plasschaert A. J. M. (1985) A new method for matching tooth colors with color standards. J. dent. Res. 64, 837-841. 
Flock S. T., Wilson B. C. and Patterson M. S. (1987) Total attenuation coefficient:s and scattering phase functions of tissues and phantom materials at $633 \mathrm{~nm}$. Med. Phys. 14, $835-841$.

Harris F. S. Jr, Shermann G. C. and Morse F. L. (1967) Experimental comparison of scattering of coherent and incoherent light. IEEE Trans. Antennas Propagation AP-15, 141-147.

Henyey L. G. and Greerstein J. L. (1941) Diffuse radiation in the galaxy. Astrophys. J. 93, 70-83.

van de Hulst H. C. (1957) Light Scattering by Small Particles. Dover Publications (1981), New York.

Jacques S. L., Alter C. A. and Prahl S. A. (1987) Angular dependence of $\mathrm{HeNe}$ laser light scattering by human dermis. Lasers Life Sci. I 4, 309-333.

Marchesini R., Bertoni A., Andreola S., Melloni E. and Sichirollo A. E. (1989) Extinction and absorption co- efficients and scattering phase functions of human tissues in vitro. Appl. Opt. 28, 2318-2324.

Meador W. E. and Weaver W. R. (1979) Diffusion approximation for large absorption in radiative transfer. Appl. Opt. 18, 1204-1208.

O'Brien W. J. (1988) Fraunhofer diffraction of light by human enamel. $J$. dent. Res. 67, 484-486.

Parsa P., Jacques S. L. and Nishioka N. S. (1989) Optical properties of rat liver between 350 and $2200 \mathrm{~nm}$. Appl. Opt. 28, 2325-2330.

Spitzer D. and ten Bosch J. J. (1975) The absorption and scattering of light in bovine and human dental enamel. Calc. Tiss. Res. 17, 129-137.

Zijp J. R. and ten Bosch J. J. (1991) Angular dependence of $\mathrm{HeNe}$-laser light scattering by dentine, a comparison of experiment and theory. To be published. 